\title{
Using the CES-D with custodial grandmothers: cross-validation and convergent validity.
}

\author{
Will Merchant ${ }^{1 *}$, Gregory Smith ${ }^{2}$, Bert Hayslip $\mathbf{J r}^{3}$ \\ ${ }^{1}$ The University of Northern Colorado, USA \\ ${ }^{2}$ Kent State University, USA \\ ${ }^{3}$ University of North Texas, USA
}

\begin{abstract}
Objective: This study is the first to analyze the factor structure of the Center for Epistemological Studies Depression (CES-D) Scale with custodial grandmothers involving both cross validation and convergent validity analyses.

Method: Cross validation was accomplished with two different samples of custodial grandmothers $(\mathrm{GCM})$ to calibrate $(n=733$; Average Age $=52)$ and then validate $(n=343$; Average Age=52.5) the model.

Results: Radloff's originally proposed four factor model (Depressed Affect, Well-Being, Somatic Symptoms, and Interpersonal Problems) was found to best fit the data for both calibration ( $R M S E A=0.049)$ and validation samples $(R M S E A=0.050)$. The construct validity of the four CES-D factors was supported by the correlations observed between these factors and conceptually related psychosocial measures.

Conclusion: four CES-D factors as proposed are psychometrically sound when applied to custodial grandmothers and that each factor contributes unique and meaningful information in its own right.
\end{abstract}

Keywords: Epistemological studies, Depression, Cross validation, Convergent validity.

Accepted on September 07, 2017

\section{Introduction}

Recent changes in social service policy such as decreased availability of foster care, increased welfare caseloads and a preference for custodianship to fall within familial lines, have increased the degree to which grandparents become the primary care providers for their grandchildren due to problems (e.g. divorce, incarceration and incompetence) experienced by the grandchild's birth parents. This growth in care distribution has resulted in approximately 937,700 grandparent households currently responsible for grandchild care. Within this configuration, referred to as "custodial" or "skipped generation" grand families, the custodial grandmother (CGM) is almost always the primary care giver for the grandchild.

Due to the above reasons for which custodial grand parenting arises, it is not uncommon for the duration of this care to exceed five or more years. As the physical, emotional and social challenges of caregiving accumulate, it is likely that psychological stress will be experienced as well. Abundant research has documented an elevated risk for depressive symptoms among family caregivers to older adults, and this phemonena has similarly been found within national samples of CGMs [1-4]. Yet, little research has been conducted to examine the psychometric properties of instruments that 1 are commonly used to assess depressive symptoms among custodial grandparents.

The Center for Epidemiological Studies Depression Scale (CES-D) is a psychometrically sound measure of depressive symptoms that was designed to identify depression among the general population which has been widely used in studies of informal family caregivers. The CES-D contains 20 items assessing the frequency of typical depressive symptoms over the span of a week [5]. Items are all answered within the context of "in the past week how often did you feel..." and include such statements as "fearful", "you could not get going", "lonely". Total scores range between 0 and 60 with scores of 16 or higher indicating significant psychological distress [5]. The basic psychometric properties of the CES-D are robust and hold across general population subgroups, and this instrument is suitable for use in Black and White Englishspeaking American populations of both sexes within a ride range of age and SES [6].

Our goal in the present study is to evaluate the appropriateness of the CES-D for specific use with CGMs. To this end, we conduct a cross validation analysis to uncover the most appropriate factor structure for eventual use with this population. We also inspect the basic psychometric properties of this instrument with CGMs, and examine the convergent 
validity and overall meaningfulness of the identified CESD factors by correlating them with measures of conceptually related constructs.

While the CES-D is often used to produce scores indicating the degree to which an individual is experiencing depressive symptoms, the structure of the items on this measure also has the potential to be reduced to varying subscales and factors. In her initial publication of the CES-D, Radloff [5] suggested a four factor structure after conducting an exploratory factor analysis on the original 20 items, based on the "eigenvalue greater than one" guideline. This procedure was conducted across three samples, all of which revealed the same factor structure and exhibited high correlation across parallel factors between samples. This four factor structure breaks apart the predominantly used one factor model of depression into the subscales of Depression (i.e., Depressed Affect, Well-Being, Somatic Symptoms and Interpersonal Problems [5]. The 20 belonging to the CES-D are shown in Figure 1, according to Radloff's break down. These items primarily measure affective and somatic aspects of depression.

As described by Shafer [7], identifying the factor structure of depression symptom measures like the CES-D and determining if these factors are the same across subgroups has important implications for assessment, treatment and theory. For example, individuals with different symptom profiles are likely to have different prognoses and may require different treatment. Thus, it is important to determine whether subgroups can be distinguished on the basis of specific depression symptom factors. It is equally important to test various aspects of a theory of depression on the basis of different symptoms that have been operationalized as specific depression symptom factors [8]. It is further possible that different depressive symptom factors may be related to specific genetic variables within a given population and those different neural substrates may be associated with diverse symptom factors. There is also growing evidence that different subgroups (e.g. gender, race/ethnicity, patent status) have different mean levels of symptomatology in terms of specific symptom factors on depression measures, and that specific depression symptom factors within a test have different patterns of correlation with external variables [7].

Although many prior studies have largely confirmed Radloff's four factor model for the CESD across a wide variety of populations [7], others have supported alternative structures such as two and three factor models [5,9-12]. Research has also indicated that for some populations, a simpler three factor model using only depression, anxiety and interpersonal problems item groupings may be better suited. For example, Chapleski, Lamphere, Kaezynski, Litchenberg and Dwyer found this alternative three factor model to perform better with American Indian Elders. Minkler et al. [13] also found a simpler factor structure to be a better fit statistically and conceptually for older African American men and women in which depression and Somatic Symptoms were found to define one factor rather than two.

Several studies have also been conducted to examine the factor structure of the CES-D as it applies specifically to family caregivers. O'Rourke [14] tested the validity of the four factor model among older adults providing care for familial dementia patients, finding it to fit their observed data well. Roth et al. [15] also tested the four factor model with the caregivers of dementia patents and not only found it to be a good fit as well, but also found this factor structure to be consistent across ethnic groups.

To our knowledge, only one prior study has examined the factor structure of the CES-D with the unique family caregiving population of grandparents raising grandchildren, despite the fact that it has been widely used by researchers

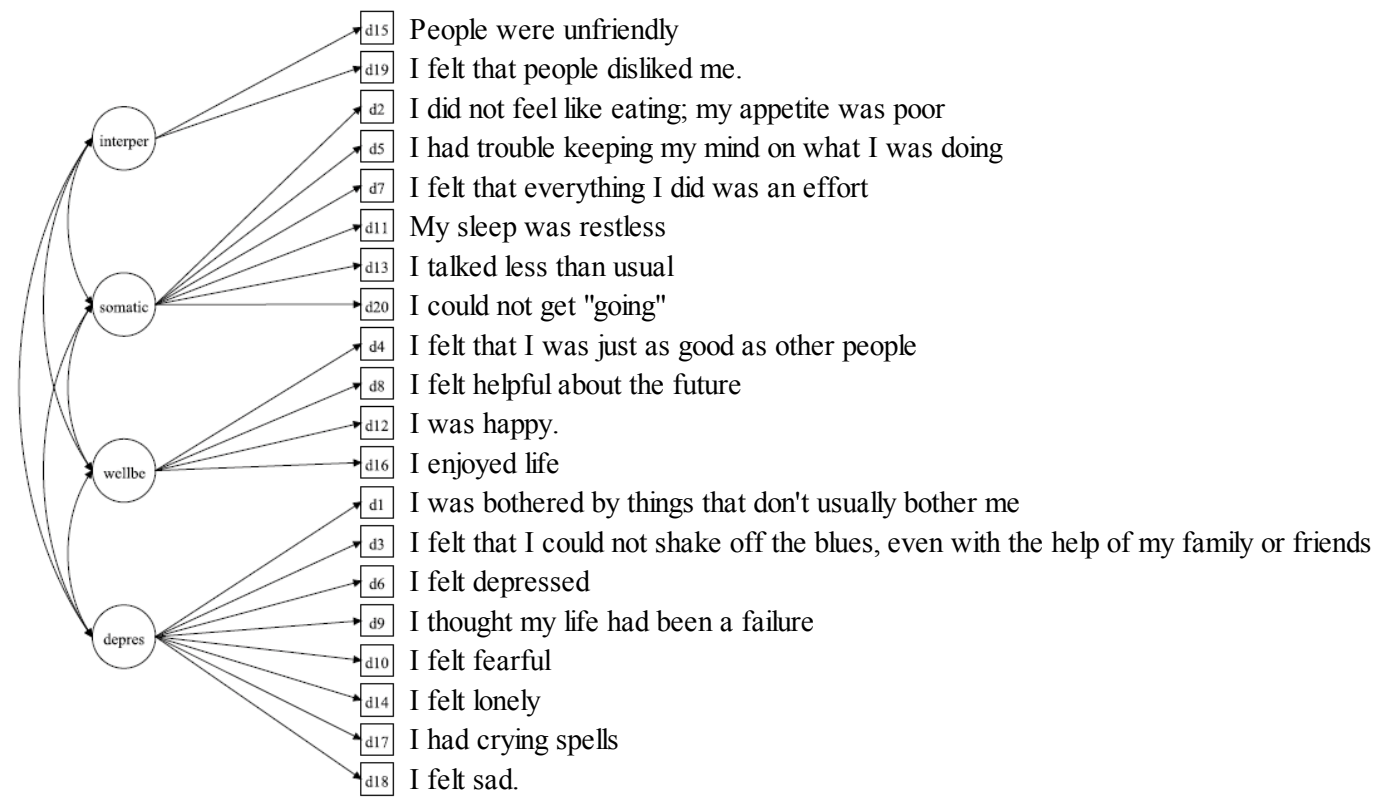

Figure 1. The 20 belonging to the CES-D. 
to investigate the level of depressive symptoms reported by these caregivers $[4,13,16,17]$. However, the study conducted by McCallion and Kolomer [18] differs substantially from the present investigation in several ways. Their sample was restricted to African American participants recruited from the boroughs of New York City and they broadly defined "grandmother carer" to include any self-defined primary carer who was "at least one generation removed from the child's own parent(s) (p. 329). In contrast, all of our participants are "true" CGMS who were recruited across the 49 contiguous states. Thus, findings from the present study are far more likely to generalize to the overall population of US CGMs.

There are also important methodological differences between the two studies. First, McCallion and Kolomer [18] used a sequence of (a) confirmatory factor analysis (with orthogonal rotation) of Radloff's [5] original four factor model, which did not fit the data well; (b) a subsequent principal components exploratory factor analysis (with varimax rotation), which fit the data better but differed from considerably from Radloff's model; and (c) a confirmatory factor analysis of the newly derived model from step $b$, which fit the data reasonably well. In the present study, we use a more rigorous structural equation modeling (SEM) approach where the four competing models of the CES-D factor structure shown in Figure 2 are compared to one another (Figure 2).

The one-factor model shown in Figure 1 simply allows all 20 items to load on a single latent construct. The twofactor model distinguishes the 4 reverse-scored positive affect items into a separate Well-Being factor from the remaining 16 depressive symptom items. The three-factor model elaborates on the two-factor model, with 2 items from the 16 depressive symptom items in the two-factor model designated as a third factor for interpersonal problems. The four-factor model is identical to CES-D factor structure proposed by Radloff [5] and includes Depressed Affect (7 items), Well-Being (4 items), Interpersonal Problems (2 items) and Somatic Symptoms (7 items). Our testing of these alternative models provides support for an optimal model not only through acceptable fit to the data, but also by the rejection of competing models [19].

Another methodological distinction of the present study, is that it represents the first attempt to cross-validate the CES-D factor structure with any family caregiver population whatsoever. Along these lines, it should be noted that McCallion and Kolomer [18] did not employ independent samples for each of their analytic steps described above, thereby capitalizing on chance due to fitting the idiosyncrasies of the sample data [19]. In contrast, we first test the four competing models in Figure 2 with a calibration sample of 733 CGMs and then cross validate the best fitting model with an independent validation sample of 343 CGMs. This approach insures that the best fitting model is not specific to a given sample. We hypothesize that Radloff's [5] proposed four factor model (Figure 1) will show superior fit compared to the three competing models in our calibration sample, and we further expect this model to similarly show good fit in our validation sample of CGMs. Our use of CFA also allows us to examine systematically if the magnitude of factor loadings is equivalent between these two independent samples [7].

A final contribution of the present study is that, to the best of our knowledge, it is pioneering with respect to examining if any of the specific depressive symptom factors found within the CES-D correlate meaningfully with relevant

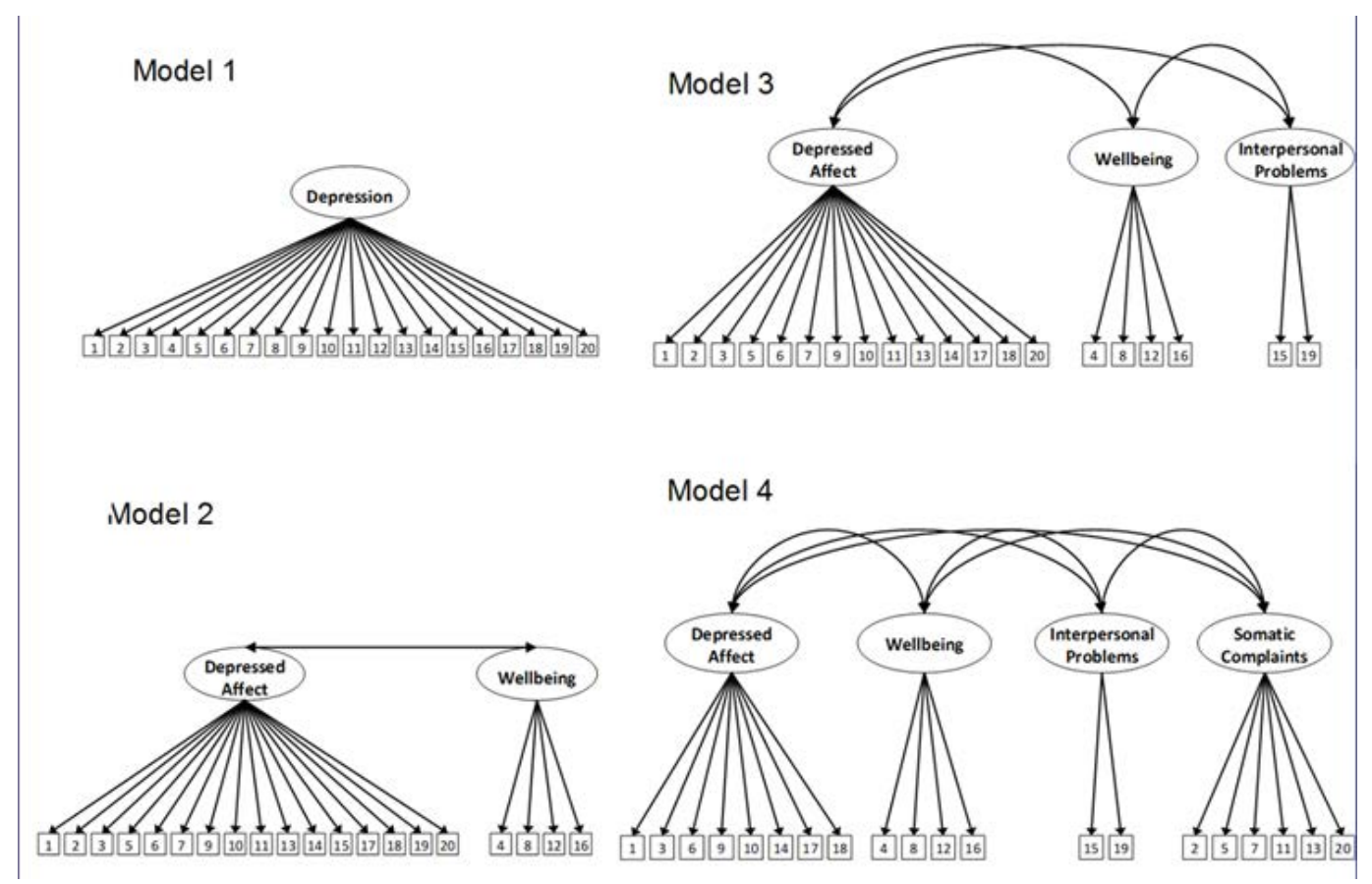

Figure 2. Competing models. 
Citation: Merchant W, Smith G, Hayslip Jr B. Using the CES-D with custodial grandmothers: cross-validation and convergent validity. $J$ Ment Health Aging. 2017;1(1):1-9.

constructs. This is an important aim because, even though there has been generally strong support for the Radloff's [5] four factor structure of the CES-D, no formal subscales exits for this instrument [7]. Consequently, the CES-D is used almost exclusively to obtain global depressions scores while potentially valuable information regarding symptom factors is overlooked. To address this gap in the literature, we will correlate the CES-D factors that are identified by means of our CFA analyses with external variables that align conceptually with Radloff's [5] proposed factors. Specifically, we expect the following correlations involving CES-D factors to be statistically significant: Depressed Affect with anxiety; Positive Affect with subjective well-being, Interpersonal Problems inversely with perceived social support and Somatic Symptoms with self-rated physical health status.

\section{Materials and Methods}

\section{Participants}

We defined CGM as the primary care provider for at least one grandchild, without involvement in caregiving from that child's birth parents. Our sample consisted of a total of 733 CGM participants in the calibration sample and 343 in the validation sample. All 1076 participants were asked to complete the CES-D, and provide relevant demographic data.

Table 1. Key sociodemographic information for study samples.

\begin{tabular}{|c|c|c|c|c|}
\hline \multirow[t]{2}{*}{ Characteristic } & \multicolumn{2}{|c|}{$\begin{array}{c}\text { Calibration } \\
\text { Sample }(n=733)\end{array}$} & \multicolumn{2}{|c|}{$\begin{array}{c}\text { Validation Sample } \\
\quad(n=343)\end{array}$} \\
\hline & $M$ or $n$ & $S D$ or $\%$ & M or $n$ & $S D$ or $\%$ \\
\hline Age (Grandmother) & 56.0 & 8.1 & $56.45 \%$ & 8.22 \\
\hline \multicolumn{5}{|l|}{ Ethnicity } \\
\hline Caucasian & 367 & $50.1 \%$ & 152 & $44.3 \%$ \\
\hline African American & 366 & $49.9 \%$ & 149 & $43.4 \%$ \\
\hline Hispanic/Latino & & & 38 & $11.1 \%$ \\
\hline Other & & & 4 & $1.2 \%$ \\
\hline \multicolumn{5}{|l|}{ Education } \\
\hline Less than high school & 123 & $16.8 \%$ & 46 & $13.4 \%$ \\
\hline High school graduate or GED & 205 & $28.0 \%$ & 64 & $18.7 \%$ \\
\hline Some college & 260 & $35.5 \%$ & 152 & $44.3 \%$ \\
\hline Bachelor's degree & 95 & $13.0 \%$ & 44 & $12.8 \%$ \\
\hline Graduate or professional degree & 50 & $6.8 \%$ & 21 & $6.1 \%$ \\
\hline Missing & & & 16 & $4.7 \%$ \\
\hline \multicolumn{5}{|l|}{ Family income } \\
\hline Under $\$ 15,000$ & 232 & $31.7 \%$ & 64 & $18.7 \%$ \\
\hline$\$ 15,000-\$ 75,000$ & 445 & $60.3 \%$ & 180 & $52.5 \%$ \\
\hline$\$ 75,000$ or more & 28 & $3.8 \%$ & 42 & $12.2 \%$ \\
\hline Missing & 28 & $3.8 \%$ & 57 & $16.6 \%$ \\
\hline \multicolumn{5}{|l|}{ Current Marital Status } \\
\hline Married & 351 & $47.9 \%$ & 132 & $38.5 \%$ \\
\hline Not Married & 382 & $52.0 \%$ & 211 & $61.5 \%$ \\
\hline \multicolumn{5}{|l|}{ Current Employment Status } \\
\hline Working & 202 & $27.6 \%$ & 145 & $42.3 \%$ \\
\hline Not Working & 531 & $72.4 \%$ & 198 & $57.7 \%$ \\
\hline
\end{tabular}

Key socio-demographic characteristics of these two samples are shown in Table 1, where it can be seen that two samples were nearly equivalent with respect to chronological age but differed slightly in other respects.

Participants in the calibration sample $(n=733)$ were recruited for an NIH funded study of stress and coping among custodial grandparents that employed a combination of convenience-based (e.g. social service agencies; Internet, radio, TV and newspaper ads) and probability-based (random recruitment letters) sampling methods. The mean age of CGMs in the calibration sample was $56.1(S D=8.1)$. Because of targeted sampling procedures, the racial distribution of this sample was roughly composed of half Caucasian and half African American participants.

Among this sample, 48\% were married, 14\% widowed, 7\% separated, 22\% divorced, 9\% single (never married), and $<1 \%$ living with a partner. The largest percentage of grandmothers had received some college education (36\%). Twenty eight percent reported being high school graduates and $17 \%$ indicated having dropped out of public school. The remaining participants (19\%) had earned at least a college degree, and some with degrees beyond undergraduate. Forty eight percent were currently living in urban environments, $19 \%$ in suburban and 33\% in rural locations. Most CGMs reported family incomes within the $\$ 15,000$ to $\$ 75,000$ per year range $(63 \%)$. The majority of CGMs were working either part or full time at the time of the interview (45\%). Fifty nine percent of grandchildren were between the ages of 4 and $10,28 \%$ were between the ages of 11 and 14 , with the remaining $13 \%$ being 15 or older.

Grandmothers in the validation sample $(n=343)$ were enrolled in a NIH funded study co-directed by the second and third authors which examined the efficacy of several psychosocial interventions on both CGM and grandchild well-being. On average, they were approximately the same age $(56.45, S D=8.22)$ as the calibration sample. The ethnic make up for this sample contained two additional categories (Hispanic/Latino, Other) which allowed for a slightly different distribution than with the calibration sample (Caucasian, 44.3\%; African American, 43.4\%; Hispanic/Latino, 11.1\%, Other, $1.2 \%$ ). Close to half of the CGMs in this sample had at least some college experience $(44.3 \%), 16.7 \%$ had a high school diploma or equivalent, $13.4 \%$ did not complete high school and $12.8 \%$ had a bachelor's degree and $6.1 \%$ had a graduate of professional degree. Most CGMs reported family incomes between $\$ 15,000$ and $\$ 75,000$ per year $(52.5 \%)$, $18.7 \%$ reported less than $\$ 15,000,12.2 \%$ reported more than $\$ 75,000$ annually, and $16.6 \%$ chose not to answer. The majority of grandmothers were not married $(61.5 \%)$ and not working $(57.7 \%)$ at the time of the interview. For the validation sample, CES-D data analyzed here involved that collected from each CGM at the initial (pre-intervention) occasion of measurement (Table 1). 


\section{Results and Discussion}

Comparisons between the four competing models depicted in Figure 1 with data obtained from the calibration sample are summarized in Table 2.

\section{Model 1}

Apart from the fact that all factor loadings associated with this model were statistically significant, none of the other indicators of model fit were within the desired range ( $R M S E A=0.079, C F I=0.846, T L I=0.876$ and $S R M R=0.059$ ).

\section{Model 2}

The $\Delta \chi_{\mathrm{SB}}^{2}$ test between this model and Model 1 was significant $\left(\Delta \chi_{\mathrm{SB}}^{2}=317.04, d f=1, p<0.01\right)$. This model performed better than Model 1 with respect to all fit indices, with some of these indices falling within the established ranges of good fit ( $C F I=0.909, T L I=0.920$ and $S R M R=0.045$ ). However, the RMSEA (0.061) was not within the accepted range of being less than 0.05. All indicators remained significant in their loading onto their designated factors. Although Model 2 showed improvement over Model 1 in all of the fit indices, there was still potential for improvement.

\section{Model 3}

The $\Delta \chi_{\mathrm{SB}}^{2}$ test between this model and Model 1 was significant $\left(\Delta \chi_{\mathrm{SB}}^{2}=124.40, d f=2, p<0.01\right)$. This model also performed better on all fit indices, than did Model 2 although RMSEA was close, it still remained somewhat outside of the desired less than 0.05 range (RMSEA $=0.053, C F I=0.933$, $T L I=0.935$ and $\mathrm{SRMR}=0.039$ ). All indicators remained significant in their loading onto their designated factors.

\section{Model 4}

A $\Delta \chi_{\$}^{2}$ test was conducted to analyze the significance of the difference between this model and Model 3. The comparison was significant indicating dissimilarity of fit $\left(\chi^{2}=56.56, d f=1, p<0.05\right)$. The RMSEA for Model 4 fell to within the desired range of less than 0.05 , further indicating improved model fit in comparison to Model 3. The remaining indices all improved as well in comparison to Model 3, further supporting the superior of this Model $4\left(\chi^{2}=448.34\right.$, $d f=65, p<0.01 ; R M S E A=0.049, C F I=0.943 ; T L I=0.942$ and $S R M R=0.037$ ). The factor loadings of all items onto their respective factors were also statistically significant and did not change in strength in comparison to the Model 3.

\section{Cross validation model}

With the above establishment of the four-factor model using the calibration sample, this solution was then tested on the validation sample (Table 2). Data from the validation sample yielded similar model fit with all fit-indices within their desired range $\left(\chi^{2}=297.24, d f=161, p<0.01 ; R M S E A=0.050\right.$, $C F I=0.943, T L I=0.952$ and $S R M R=0.041$ ). This fit allowed for the next step of the cross validation in which invariance was tested between the calibration and validation samples at the configural and scalar levels.

Configural $\left(\chi^{2}=537.13, d f=324, p<0.01 ; R M S E A=0.045\right.$, $C F I=0.962, \quad T L I=0.955$ and $S R M R=0.041)$ and scalar $\left(\chi^{2}=510.87, d f=308, p<0.01 ; R M S E A=0.047, C F I=0.961\right.$, $T L I=0.956$ and $S R M R=0.041$ ) models each exhibited excellent fit as indicated by the fit indices used above. Additionally, the Chi-square difference test between configural and scalar was non-significant suggesting that both the factor structure and factor loadings were consistent between the two samples with all factor loadings remaining significant and no observed cross-loadings.

\section{Correlational findings}

Zero order correlations between each of the CES-D symptom factors associated with Model 4 and the relevant external variables are summarized in Table 3. As expected, all correlations were statistically significant. Correlations of the highest magnitudes involved Somatic Symptoms with anxiety (0.63) and Depressed Affect with anxiety (0.58). For the purpose of the correlational analyses only, the items comprising the CES-D well-being factor were scored such that higher scores reflected higher levels of positive emotional well-being. This was done in order to better facilitate interpretation of the involved correlations. The variable subjective well-being correlated moderately with the symptom factors of Depressed Affect (-0.42), Positive Affect $(0.43)$ and Somatic Symptoms (-0.40). Moderate correlations

Table 2. Summary of the model fit indices for calibration and validation samples.

\begin{tabular}{|c|c|c|c|c|c|c|}
\hline Model & $x^{2}$ & df & RMSEA & CFI & TLI & SRMR \\
\hline \multicolumn{7}{|l|}{ Test of different CES-D factor structures } \\
\hline \multicolumn{7}{|l|}{ (Calibration Sample, $n=733$ ) } \\
\hline One Factor & 945.46 & 170 & 0.079 & 0.846 & 0.876 & 0.059 \\
\hline Two Factor & $628.50 * * *$ & 169 & 0.061 & 0.909 & 0.92 & 0.045 \\
\hline Three Factor & $504.90 * * *$ & 167 & 0.053 & 0.933 & 0.935 & 0.039 \\
\hline Four Factor & 448.34 *** & 164 & 0.049 & 0.943 & 0.942 & 0.037 \\
\hline \multicolumn{7}{|l|}{ (Validation Sample, $\mathrm{n}=343$ ) } \\
\hline Four Factor & 297.24 *** & 161 & 0.05 & 0.952 & 0.943 & 0.041 \\
\hline \multicolumn{7}{|l|}{ Invariance tests } \\
\hline Adjusted difference test between configural and scalar & $26.26, p=0.06$ & 16 & .. & .. & .. & .. \\
\hline
\end{tabular}

${ }^{* * *} p<0.01 . X^{2}=$ Chi-Square Test (i.e., Minimum Fit Function); RMSEA: Root-Mean-Square-Error of Approximation; CFI: Comparative Fit Index; TLI: Tucker Lewis Index; SRMR: Standardized Root-Mean-Square Residual Index; WRMRI Weighted Root Mean Square Residual 
Citation: Merchant W, Smith G, Hayslip Jr B. Using the CES-D with custodial grandmothers: cross-validation and convergent validity. $J$ Ment Health Aging. 2017;1(1):1-9.

were also observed for Positive Affect with anxiety (-0.42) and for Somatic Symptoms and self-rated health -0.38 . All remaining correlations were modest in magnitude despite reaching statistical significance (Table 3).

Additional findings regarding the psychometric properties of the four CFA symptom factors identified through the CFA analyses described above are summarized in Table 4. First, the Cronbach alpha reliability estimates for the items associated with each symptom factor were found to be acceptable except within both samples except for those involving the Interpersonal Problems symptom factor. Second, the zero order correlations between each CES-D symptom factor are presented separately for both the calibration and validation samples. For both samples, all correlations were statistically significant. In particular, two correlations within the calibration sample were quite large in magnitude: Depressed Affect with Positive Affect (-0.82) and Depressed Affect with Somatic Symptoms (0.93). These same correlations were of the greatest magnitude for the validation sample, but at lesser magnitudes ( -0.65 and 0.75 , respectively). Third, Table 4 also shows for both samples, the zero order correlations between each of the four symptom factors and the total CES-D score across all 20 items. All correlations across both samples were statistically significant and were very high in magnitude except for the Interpersonal Problems factor (i.e., 0.67 in calibration sample and 0.57 in validation sample) (Table 4).

\section{Discussion}

Our overall aim in this study was to determine the best fitting factor structure of the CES-D as it applies to the unique family caregiving population of CGMs. Even though past CFA studies have typically provided evidence in support

Table 3. Correlations between CES-D factors and convergent variables.

\begin{tabular}{|l|c|c|c|c|}
\hline & $\begin{array}{c}\text { Social } \\
\text { Support }\end{array}$ & Anxiety & Health & $\begin{array}{c}\text { Positive } \\
\text { affect }\end{array}$ \\
\hline Depressed Affect & -0.25 & 0.58 & -0.3 & -0.42 \\
\hline Well-Being & 0.28 & -0.42 & 0.26 & 0.43 \\
\hline Somatic Symptoms & -0.26 & 0.63 & -0.38 & -0.4 \\
\hline Interpersonal Problems & -0.25 & 0.29 & -0.19 & -0.2 \\
\hline
\end{tabular}

All correlations significant at $p<0.05$. The items comprising the CES-D positive affect factor were scored such that higher scores reflected higher levels of positive emotional well-being of Radloff's [5] originally proposed four factor structure [7], only one other study to date has involved CGMs as the population of interest. Although McCallion and Kolomer [18] ended up identifying a four factor model for the CESD-D in their study, they found a considerable numbers of crossloadings between these factors and one item ("I feel that everything I did was an effort") did not load onto any factors at all. Thus, the factors found by McCallion and Kolomer were essentially dissimilar from those observed by Radloff [5] and numerous other investigators regarding the CES-D.

In contrast to McCallion and Kolomer [18], we found Radloff's exact four factor structure to best fit the data obtained from both our calibration and validation samples. In each sample, all items loaded at statistically significant levels precisely as specified by Radloff [5] and no cross loadings whatsoever emerged. In addition, there we no significant differences between our calibration and validation samples with respect to the magnitudes of these factor loadings.

The generalizability of our findings is further enhanced by the fact that these two samples were obtained from multiple states and were quite diverse in terms of key sociodemographic characteristics. Also, it is noteworthy that our calibration and validation samples were gathered for two different purposes, with the calibration CGMs agreeing to participate in survey study only whereas the validation CGMs participated in a randomized control trial. This distinction between the two samples is important in light of concerns among researchers that family caregivers who enroll in interventions may be dissimilar from their non-help-seeking peers [20].

There are several potential reasons why our findings with the present samples of CGMs were consistent with Radloff's [5] four factor structure for the CES-D, while those reported by McCallion and Kolomer [18] were not. For instance, their sample consisted entirely of African American participants from New York City and it included caregivers other than just grandmothers. In contrast, our sample was racially/ ethnically diverse and it contained grandmothers only. Thus, we believe that our samples in the present study were far more representative of the overall U.S. population of CGMS.

That our findings in support of Radloff's original [5] four

Table 4. Inter-factor correlations and internal reliability.

\begin{tabular}{|c|c|c|c|c|c|c|}
\hline \multicolumn{7}{|c|}{ Inter-factor Correlations and internal reliability - Calibration Sample } \\
\hline & Depressed Affect & Well-Being & Somatic Symptoms & Interpersonal Problems & Total CES-D Score & $\alpha$ \\
\hline Depressed Affect & 1 & & & & 0.92 & 0.87 \\
\hline Well-Being & -0.82 & 1 & & & -0.81 & 0.71 \\
\hline Somatic Symptoms & 0.93 & -0.74 & 1 & & 0.9 & 0.74 \\
\hline Interpersonal Problems & 0.66 & -0.53 & 0.67 & 1 & 0.67 & 0.66 \\
\hline \multicolumn{7}{|c|}{ Inter-factor Correlations and internal reliability - Validation Sample } \\
\hline Depressed Affect & 1 & & & & 0.85 & 0.89 \\
\hline Well-Being & -0.65 & 1 & & & -0.72 & 0.78 \\
\hline Somatic Symptoms & 0.75 & -0.51 & 1 & & 0.83 & 0.74 \\
\hline Interpersonal Problems & 0.44 & -0.29 & 0.43 & 1 & 0.57 & 0.63 \\
\hline
\end{tabular}

All correlations significant at $p<0.05 . \alpha=$ Cronbach's alpha. The items comprising the CES-D Positive Affect factor were scored such that higher scores reflected higher levels of positive emotional well-being 
factor structure differ from McCallion and Kolomer's [18] findings may also be due to key methodological differences between the two studies. Whereas they conducted several different types of analyses that all involved a single sample, we followed the approach of comparing four competing CFA models which has become the standard in this line of research [7]. Moreover, we uniquely confirmed our findings across two different samples.

In addition to cross-validating Radloff's [5] factor structure of the CES-D, we also examined key psychometric properties of these four symptom factors across our two separate samples of CGMs. Such analyses are important because, even though no formal symptom subscales currently exist for the CES-D, the items associated with each symptom factor (Figure 2) would presumably be used by clinicians and researchers alike to measure each of the four CES-D symptom factors, respectively [7]. In this regard, we found the internal consistency values as measured by Cronbach's alpha for each symptom factor to be nearly identical across both the calibration and validation samples, with all symptom factors showing acceptable alpha values (i.e., $\geq 0.70$ ), except for the Interpersonal Problems factor. The less than ideal Cronbach alpha values observed for this particular symptom factor are likely due to the fact that it is measured by only two items, which is problematic given that the reliability of a scale's items is generally underestimated when the number of items is small [21]. Because the CES-D is the only widely used measure of depressive symptoms at this time to contain an interpersonal problems factor [7], an important future goal is the development of additional items that uniquely capture this important aspect of depression.

From a psychometric perspective, we also examined within both the calibration and validation samples the degree to which each of Radloff's [5] symptom factors correlated with each other as well as with the CES-D total score. In both samples, the highest correlations between the symptom factors and CES-D total score involved the Depressed Affect factor and the Somatic Symptoms factor. In turn, this is in line with Shafer's [7] meta-analysis of the factor structures of the four major depression questionnaires which found that these two symptom factors are common across the Beck Depression Inventory [22], the Hamilton Rating Scale for Depression [23], Zung Self-Rating Scale [24] and the CES-D alike.

The primacy of the Depressed Affect and Somatic Symptoms factors is further indicated by the finding that these two symptom factors correlated the highest with each other in both our calibration (0.93) and validation samples (0.75). In contrast, the Interpersonal Problems factor showed the lowest inter correlations with both the CES-D total score and the other three symptom factors in both samples. It is noteworthy that this particular symptom factor was found to be unique to the CES-D in Shafer's [7] meta-analysis of the four major depressive questionnaires. This reinforces the recommendation stated above that further development of the Interpersonal Problems symptom factor is warranted.
Our correlational findings within both the calibration and validation samples additionally mirror Shafer's [7] conclusion that "the General Depression factor found in all the tests is probably the most appropriate and pure measure of depressive symptom severity" (p. 137).

We also set out in the present study to examine if the depressive symptom factors on the CES-D were related to conceptually-related external variables in our calibration sample. This enabled us to not only conduct a preliminary assessment of each factors potential construct validity, but also provide evidence to support the future use of these symptom factors by clinicians and researchers alike. This is a major contribution of the present study given that, to the best of our knowledge, similar analyses have yet to be conducted with CGMs or any other target population for that matter. If these symptom factors are, in fact, associated with other key variables then their potential usefulness in informing theory, identifying population subgroups and serving as specific intervention outcomes is indicated $[7,8]$.

The external variables that we examined included measures of perceived emotional support, self-reported anxiety symptoms, and self-rated physical health. Each of these variables was found to correlate significantly with all four CES-D symptom factors. The Depressed Affect factor of the CES-D had the greatest correlation with Anxiety $(r=0.58)$, which is not surprising given that anxiety and depression are highly interrelated [25]. Indeed, depression and anxiety often coexist in later life [26] and depression with anxiety symptoms or with a comorbid anxiety disorder is a more severe illness in older adults than depression without anxiety, in terms of course and outcome [27]. The remaining correlations involving Depressed Affect were moderate, negative, and coincided with the prediction that general depression would be negatively related to perceived social support, better self-rated health, and subjective positive wellbeing given that each of these variables has been found to be inversely associated with depressive symptoms in the general population [28-30].

Our finding that the Positive Affect factor of the CES-D correlated most highly with subjective well-being $(r=0.43)$ supports the convergent validity of this symptom factor given that its corresponding items (Figure 1) focus specifically on such positive mood states as happiness, enjoyment, and hopefulness. However, the Positive Affect symptom factor also showed correlations of a similar magnitude with anxiety which corresponds to recent genetic studies which point to genetic linkages between depressive symptoms, neuroticism and subjective well-being [31]. Understanding the role of possible genetic predispositions in mood disorders has been a major goal within the NIMH [8] strategic plan for mood disorders research.

The Somatic Symptoms factor of the CES-D had its greatest correlation with Anxiety $(r=0.63)$, which is understandable given that several of the CES-D items 
Citation: Merchant W, Smith G, Hayslip Jr B. Using the CES-D with custodial grandmothers: cross-validation and convergent validity. $J$ Ment Health Aging. 2017;1(1):1-9.

corresponding to this factor such as reduced appetite, fatigue, restless sleep, and difficulty concentrating are also common symptoms of anxiety [25]. This Somatic Symptoms factor of the CES-D also negatively correlated with perceived social support, self-rated health and subjective well-being, but the magnitude of these correlations was not as high that involving anxiety. In this respect, it is noteworthy that the CES-D Somatic Symptoms factor includes motivational items ("could not get going"; "everything is an effort") that are not present among the somatic symptoms items on other major depression questionnaires [7]. It is possible then, that its correlation with such variables as social support and subjective well-being may be largely attributable to the presence of these motivational items.

Although the CES-D Interpersonal Problems factor correlated with all four of the external variables, the low magnitude of theses correlations suggests that only a small amount of the variance within each is associated with this symptom factor. This further reinforces the concerns raised above that this symptom factor, which is unique to the CES-D, may be currently insufficiently measured by just two items. Our findings also point to the possibility that interpersonal problems are not symptomatic of depression per se. According to DSM-5 criteria, for example, impairments in the social realm and other important areas of functioning are the result of depressive symptoms [32].

It is important to acknowledge several limitations associated with the present study. One shortcoming is that we did not test the CES-D factor by CGM race/ethnicity and custodial grandfathers were not considered at all. The fact that our findings with a racially diverse sample differed from those reported by McCallion and Kolomer [18] with an African American only sample, point to possible racial differences among CGM regarding CES-D symptom factors. Another limitation is that our examination of the correlations between CES-D symptom factors and relevant external variables was restricted to the calibration sample only because these variables were not similarly collected in the validation sample. Moreover, the external variables that we examined here were from a larger study and selected for a different purpose other than investigating their relationships to CES-D symptom factors.

Despite these limitations, the findings of the present study provide convincing evidence among two different national samples of CGMs supporting the four symptom factor of the CES-D as was originally identified by Radloff [5]. Although the psychometric properties of three of these symptoms factors were found to be strong (i.e., Depressed Affect, Somatic Symptoms and Positive Affect), our results also raise several important concerns regarding the measurement properties and overall meaningfulness of the Interpersonal Problems factor that might transcend specific use of the CES-D with CGMs. Finally, we hope that the significant correlations we observed between the four CES-D symptom factors and conceptually relevant external variables will encourage clinicians and researchers in the future to explore the usefulness of these factors in identifying patient profiles, serving as treatment outcomes and facilitating theoretical understanding of depressive symptoms for all target populations, especially custodial grandmothers. With the increase of situations in which grandparents are functioning as the full-time caregivers for their grandchildren, it is important to know that the widely used CES-D can be meaningfully used with this population.

\section{Funding}

Work on this was funded by R01MH066851 awarded to the third author and 1R01NR012256 awarded to the second and third authors.

\section{References}

1. Bachman HJ, Chase-Lansdale PL. Custodial grandmothers' physical, mental and economic well-being: Comparisons of primary caregivers from low-income neighborhoods. Fam Relat. 2005;54(4):475-87.

2. Goodman CC, Silverstein M. Grandmothers raising grandchildren ethnic and racial differences in well-being among custodial and co-parenting families. J Fam Issues. 2006;27(11):1605-26.

3. Musil C, Warner C, Zauszniewski J, et al. Grandmother caregiving, family stress and strain and depressive symptoms. West J Nurs Res. 2009;31(3):389-408.

4. Smith GC, Palmieri PA, Hancock GR, et al. Custodial grandmothers' psychological distress, dysfunctional parenting and grandchildren's adjustment. Int J Aging Hum Dev. 2008;67(4):327-57.

5. Radloff LS. The CES-D scale: A self-report depression scale for research in the general population. Appl Psychol Meas. 1977;1:385-401.

6. Lewinsohn PM, Seeley JR, Roberts RE, et al. Center for epidemiologic studies depression scale (CES-D) as a screening instrument among community-residing older adults. Psychol Aging. 1997;12: 277-87.

7. Shafer AB. Meta-analysis of the factor structures of four depression questionnaires: beck, CES-D, hamilton and zung. J Clin Psychol. 2006;62:123-46.

8. National Institute of Mental Health. Breaking ground, breaking through: The strategic plan for mood disorders research (NIH publication no. 03-5121). National Institutes of Health, Washington DC 2003.

9. Hertzog C, Van Alstine J, Usala PD, et al. Measurement properties of the center for epidemiological studies depression scale (CES-D) in older populations. Psychol Assess. 1990;2:64-72.

10. Knight RG, Williams S, McGee R, et al. Psychometric properties of the center for epidemiologic studies depression scale (CES-D) in a sample of women in middle life. Behav Res Ther. 1997;35:373-80. 
11. Nguyen HT, Kitner-Triolo M, Evans MK, et al. Factorial invariance of the CES-D in low socioeconomic status African Americans compared with a nationally representative sample. Psychiatry Res. 2004;126:177-87.

12. Wong YI. Measurement properties of the center for epidemiologic studies - Depression scale in a homeless population. Psychol Assess. 2000;12:69-76.

13. Minkler M, Fuller-Thomson E, Miller D, et al. Depression in grandparents raising grandchildren: Results of a national longitudinal study. Fam Med. 1997;6(5):445-60.

14. O'Rourke N. Factor structure of the center for epidemiologic studies-depression scale (CES-D) among older men and women who provide care to persons with dementia. Int J Testing. 2005;5(3):265-77.

15. Roth DL, Ackerman ML, Okonkwo OC. The four-factor model of depressive symptoms in dementia caregivers: A structural equation model of ethnic differences. Psychol Aging. 2008;23(3):567.

16. Smith GC, Strieder F, Greenberg P, et al. Patterns of enrollment and engagement of custodial grandmothers in a randomized clinical trial of psychoeducational interventions. Fam Relat. 2016;65:369-86.

17. Szinovacz ME, DeViney S, Atkinson MP. Effects of surrogate parenting on grandparents' well-being. J Gerontol. 1999;54B:S376-88.

18. McCallion P, Kolomer SR. Depressive symptoms among African American caregiving grandmothers: The factor structure of the CES-D. J Ment Health Aging. 2000;6(4):551-70.

19. Bandalos DL, Finney SJ. Factor analysis: Exploratory and confirmatory. In: The reviewer's guide to quantitative methods in the social sciences. Hancock G, Mueller R eds. Routledge, New York. 2010;93-114.

20. Schulz R, Newsom J, Mittelmark M, et al. Health effects of caregiving: The caregiver health effects study: an ancillary study of the cardiovascular health study. Ann Behav Med. 1997;19:110-16.
21. Tvakol M, Dennick R. Making sense of cronbach's alpha. Int J Med Educ. 2011;2:53-55.

22. Beck A, Ward C, Mendelson M, et al. An inventory for measuring depression. Arch Gen Psychiatry. 1961;4:56171 .

23. Hamilton M. Development of a rating scale for depression. J Neurol Neurosurg Psychiatry.1967;23:56-62.

24.Zung W. A self-rating depression scale. Arch Gen Psychiatry. 1965;12:63-70.

25. Lovibond PF, Lovibond SH. The structure of negative emotional states: Comparison of the depression anxiety stress scales (DASS) with the beck depression and anxiety inventories. Behav Res Ther. 1995;33(3):335-43.

26. Blazer, D. Depression in late life. Springer, New York. 2002.

27. Lenze E, Mulsant B, Shear K, et al. Comorbidity of depression and anxiety disorders in later life. Depress Anxiety. 2001;14:86-93.

28. Burns R, Anstey K, Windsor T. Subjective well-being mediates the effects of resilience and mastery on depression and anxiety in a large community sample of young and middle aged adults. Aust N Z J Psychiatry. 2011;45:240-48.

29. Gray S, Hellzen O, Romild U, et al. Association between social support and depression in the general population: The hunt study, a cross-sectional survey. J Clin Nurs. 2012;21:111-20.

30. Wagner D, Short JL. Longitudinal predictors of self-rated health and mortality in older adults. Prev Chronic Dis. 2014;11:1-8.

31. Okbay A, Baselmans BM, De Neve JE, et al. Genetic variants associated with subjective well-being, depressive symptoms and neuroticism identified through genomewide analyses. Nat Genet. 2016;46:624-31.

32. Diagnostic and statistical manual of mental disorders (5th ed.). American Psychiatric Association, Washington DC. 2013.

\section{*Correspondence to:}

Will Merchant

The University of Northern Colorado

USA

Tel: (610) 499-4289

E-mail: wrmerchant@widener.edu 